\title{
Actividad in vitro de la mezcla de alcaloides de Ervatamia coronaria (Jacq) Staff. Apocynaceae sobre amastigotes de Leishmania braziliensis
}

\author{
Amanda Moreno Rodríguez, ${ }^{1}$ Jorge Robles Camargo, ${ }^{*, 2}$ Felio J. Bello García ${ }^{3}$

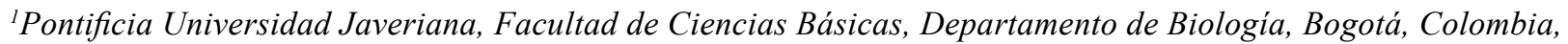 \\ ${ }^{2}$ Pontificia Universidad Javeriana, Facultad de Ciencias Básicas, Departamento de Química. Bogotá, Colombia, \\ ${ }^{3}$ Universidad del Rosario, Instituto de Ciencias Básicas, Facultad de Medicina, Bogotá, Colombia
}

\begin{abstract}
RESUMO: "Atividade in vitro da mistura de alcalóides de Ervatamia coronaria (Jacq) Staff. Apocynaceae sobre amastigotas de Leishmania braziliensis". A leishmaniose é considerada uma importante causa de morbidade e mortalidade a nível mundial, principalmente nos países tropicais. As formas cutânea e mucocutânea são causadas, entre outras espécies, por Leishmania braziliensis. Na procura de compostos leishmanicidas de origem natural, foi estudada a atividade da mistura de alcalóides de Ervatamia coronaria (Apocynaceae) contra amastigotas de $L$. braziliensis em 6 concentrações diferentes $(1,10,20,25,50$ e $100 \mu \mathrm{g} / \mathrm{mL})$. Foram tratados macrófagos de ratos da linha J774, infectados com promastigotas de L. braziliensis, com a mistura de alcalóides 1 hora após-infecção e diariamente por 3 dias sem mudança de meio. As experiências de citotoxicidade foram efetuadas sobre os macrófagos com azul tripam. Todos os cultivos foram feitos de forma triplicada e os grupos de controle não foram submetidos à mistura de alcalóides. Foi obtido que o composto adicionado exerce atividade doses/dependente sobre a parasita. No entanto, as concentrações mais altas $(50$ e $100 \mu \mathrm{g} / \mathrm{mL})$, adicionado durante 3 dias, mostraram os maiores índices de infecção, provavelmente devido a diminuição no número de macrófagos, sobre os quais não foi observado efeito tóxico do tratamento durante 24 horas $\mathrm{DL}_{50} / 24 \mathrm{~h}=233,52$ $\mu \mathrm{g} / \mathrm{mL}$. Os resultados dessa pesquisa revelaram uma nova atividade farmacológica de alcalóides da espécie Ervatamia coronaria sobre a forma amastigota de Leishmania braziliensis, com $\mathrm{IC}_{50}$ $=2,6$ e $12,4 \mu \mathrm{g} / \mathrm{mL}$ sem mostrar toxicidade sobre a célula hospedeira.
\end{abstract}

Unitermos: Leishmania, alcalóides, Ervatamia coronaria, Apocynaceae, citotoxicidade, amastigota.

\begin{abstract}
In vitro activity of the alkaloids mixture of Ervatamia coronaria (Jacq) Staff. Apocynaceae on Leishmania braziliensis amastigotes". Leishmaniasis, caused by Leishmania sp., is one of the mean reason of considerable mortality and morbidity throughout the world, especially in the tropics. Cutaneous and mucocutaneous manifestations are caused by Leishmania braziliensis, and the cutaneous form is the most common one in Colombia. In the search for antileishmanial compounds from natural sources, we studied the alkaloids mixture from Ervatamia coronaria against $L$. braziliensis at six different concentrations $(1.0,10,20,25$, 50 and $100 \mu \mathrm{g} / \mathrm{mL}$ ). Macrophages $\mathrm{J} 774$ infected with L. braziliensis were treated with alkaloids one hour, and once a day for three days, after parasitic infection and preserving the same culture medium. Cytotoxicity with trypan blue was undertaken in macrophages $\mathrm{J} 774$ by using the same concentrations. Three different cultures samples were carried out. As a control we used medium alone. The alkaloids mix showed a dose/dependent activity on amastigote, but by increasing concentrations from 50 to $100 \mu \mathrm{g} / \mathrm{mL}$ for three days, we saw a high index of infection, probably caused by cellular death. We did not see any toxic effect on macrophages $\mathbf{J} 774$ at $100 \mu \mathrm{g} / \mathrm{mL}$, $\mathrm{LD}_{50} / 24 \mathrm{~h}=233.52 \mu \mathrm{g} / \mathrm{mL}$. These results revealed a novel pharmacological activity of alkaloids from $E$. coronaria against amastigotes of L. braziliensis $\mathrm{IC}_{50}=2.6$ and $12.4 \mu \mathrm{g} / \mathrm{mL}$ without toxicity on host cells.
\end{abstract}

Keywords: Leishmania, alkaloids, Ervatamia coronaria, Apocynaceae, cytotoxicity, amastigote.

\section{INTRODUCCIÓN}

La leishmaniosis es un conjunto de enfermedades causadas por protozoos del género
Leishmania y representa una causa importante de morbilidad y mortalidad en países tropicales y subtropicales. Estos parásitos originan las enfermedades que se agrupan en: cutánea, mucocutánea y visceral. 
En Colombia la forma cutánea es la más común, representando más del 90\% de infecciones sintomáticas y es causada, principalmente, por Leishmania (Viannia) panamensis y Leishmania (Viannia) braziliensis.

El tratamiento básico consiste en la administración de stilbogluconato de sodio (Pentostam), glucantime o pentamidina. Sin embargo, existen fallas y limitaciones en el tratamiento, especialmente en las formas de leishmaniosis visceral, mucosa y cutánea difusa. Actualmente el fracaso del tratamiento se relaciona con la resistencia del parásito y la toxicidad que presenta la droga (Berman 1997). Por otro lado, se trata de medicamentos de difícil acceso por parte de las comunidades rurales que, además, presentan deficiencias nutricionales en la mayoría de casos.

Las plantas proveen una gran diversidad química y de bioactividad. Las condiciones del trópico hacen que esta región presente una alta diversidad de flora que conforma un material potencial, poco explorado científicamente; práctico y eficiente en el manejo de problemas epidemiológicos de diferentes orígenes, además de contribuir al conocimiento fitofarmacológico a través del uso de los recursos naturales y de brindar un respaldo científico del uso tradicional de las plantas por las comunidades humanas. Dentro de los metabolitos secundarios vegetales más conocidos química $\mathrm{y}$ farmaceúticamente, y usados en el campo medicinal, están los alcaloides. Estos compuestos han sido aislados de varias especies de la familia Apocynaceae, de diferentes partes de la planta: semillas, corteza, hojas y tallos, principalmente (Quintans-Júnior et al., 2007; Souza et al., 2007). La mayoría de especies de esta familia han sido usadas contra el reumatismo, resfriados y enfermedades de la piel, incluyendo infecciones (Agra et al., 2007). Se conocen 180 géneros y más de 1.600 especies cuya química presenta compuestos tipo terpenoides, lignanos, glicósidos y alcaloides, más específicamente alcaloides tipo indólico y bisindólicos (Márquez 1999; Rocha et al., 2005) que han despertado interés como anticancerígenos (vinblastina), hipertensivos (yohimbina) y específicamente la especie Peschiera australis presenta alcaloides indólicos con actividad contra Leishmania amazonensis (Delorenzi et al., 2001). Contra la misma especie de parásito se estudió la apocinácea Tabernaemontana catharinensis de la cual se trabajó con su extracto etanólico (Soares et al., 2003); y recientemente, el extracto de alcaloides de Aspidosperma ramiflorum mostró efectividad contra las formas promastigotes y amastigotes de Leishmania braziliensis (Piloto et al., 2004).

Considerando a los miembros de la familia Apocynaceae como elementos importantes en la medicina tradicional de varias comunidades de Latinoamérica, se reconocen a aquellos como agentes potenciales para el tratamiento de varias parasitemias. El objetivo de este trabajo es determinar si la mezcla de alcaloides de la especie Ervatamia coronaria (Jacq) Staff., conocida vulgarmente en Colombia como "lechoso", "sanango" y "buquecito", tiene o no actividad leishmanicida para las formas intracelulares (amastigotes) de Leishmania braziliensis en condiciones in vitro.

\section{MATERIALES Y MÉTODOS}

\section{Obtención de la mezcla de alcaloides}

La muestra de Ervatamia coronaria se obtuvo de un especimen adulto en el Jardín Botánico del Quindío, Colombia en las coordenadas específicas N 4³0’35.5" W 75³9'07.3“ en noviembre de 2004. La determinación estuvo a cargo del Herbario Nacional Colombiano y el ejemplar fue depositado con el número COL505069 del mismo herbario.

El material se secó a la sombra, y fue pulverizado en un molino industrial obteniendo $364 \mathrm{~g}$ de peso seco. Se hizo la extracción con etanol destilado $95 \%$ por maceración durante ocho días con agitación diaria. Después de la filtración de este extracto se concentró en el rotavapor y se acidificó en $500 \mathrm{~mL}$ de ácido acético $10 \% \mathrm{v} / \mathrm{v}$. Esto se mantuvo a $5{ }^{\circ} \mathrm{C}$ durante 15 horas para ser filtrada y el producto se extrajo con diclorometano para obtener el extracto ácido, el cual presentó un $\mathrm{pH}$ de 2 . La solución ácida se basificó con $\mathrm{NaOH}$ al $40 \%$ hasta $\mathrm{pH} 10$, a la que se le realizó una re-extracción con diclorometano. Tanto el extracto básico como la solución se volvieron a extraer con diclorometano y fueron llevadas a mínimo volumen. Pruebas de Dragendorff se aplicaron al extracto ácido, solución básica y extracto básico obteniendo positivo, negativo y fuertemente positivo, respectivamente. Con lo cual se escogió el extracto básico para hacer los ensayos biológicos. La cromatografía en capa delgada CCD con fase estacionaria de sílica gel y fase móvil diclorometano: metanol en proporción 9:1 y acetato de etilo en un volumen de $20 \mathrm{~mL}$ reveló sustancias de alta polaridad en colores correspondientes a compuestos de tipo alcaloide. La mezcla fue mantenida a $-20{ }^{\circ} \mathrm{C}$ hasta su uso en los ensayos celulares.

\section{Mantenimiento de cultivos celulares y parásitos}

La cepa de Leishmania braziliensis suministrada por el Centro Internacional de Física, de la Universidad Nacional se mantuvo en medio axénico NNN bifásico modificado con Schneider's y enriquecido con suero fetal bovino SFB inactivado al $10 \%$ a $26{ }^{\circ} \mathrm{C}$. Los macrófagos J774 se mantuvieron en medio RPMI 1640 con SFB al 5\%.

\section{Actividad anti-amastigote}

Macrófagos J774 se sembraron en cajas con 24 pozos, en los cuales se colocó previamente una laminilla estéril de $12 \mathrm{~mm}$ de diámetro Fischer brand microscope cover glass como soporte para la formación de la 
monocapa. Los macrófagos adheridos fueron infectados con promastigotes de L. braziliensis en relación parásito/ macrófago de 6:1, con una concentración de $10^{6}$ parásitos/ $\mathrm{mL}$. Las células infectadas se llevaron a incubación a $37{ }^{\circ} \mathrm{C}$ durante 4 días. La adición de concentraciones de la mezcla de alcaloides se hizo una hora después de la infección, y diariamente por 3 días post-infección, sin cambio de medio. Las concentraciones evaluadas fueron $1.0,10.0,20.0,25.0,50.0,100.0 \mu \mathrm{g} / \mathrm{mL}$ solubilizadas en DMSO $1.5 \%$. El control correspondió a los cultivos sin adición de la mezcla de alcaloides.

El día 4, las laminillas fueron extraídas de cada pozo y las células se fijaron con metanol puro y se colorearon con Giemsa 10\%. El número de amastigotes fue determinado haciendo el conteo de al menos, 300 células en cultivos por triplicado. Los resultados son expresados como el porcentaje de infección, proporción de infección y porcentaje de supervivencia en comparación con los controles. El porcentaje de infección, y su correspondiente proporción fueron obtenidos con el número de células infectadas/células totales por campo observado. El índice de supervivencia se calculó multiplicando el porcentaje de macrófagos infectados por el número de amastigotes por macrófago infectado.

\section{Prueba de viabilidad en macrófagos}

Macrófagos J774 se sembraron en caja de 24 pozos en una concentración de $8 \times 10^{4}$ células/pozo sin adición de alcaloides, y con las respectivas concentraciones las cuales se añadieron 24 horas después de la siembra. Pasadas 48 horas se removió el medio de cultivo y se hizo un lavado con solución salina. Se adicionaron 50 $\mu \mathrm{L}$ de medio fresco y la misma cantidad de azul tripan $0.4 \% \mathrm{p} / \mathrm{v}$ y se llevó a incubación durante 10 minutos a $37^{\circ} \mathrm{C}$ para realizar el conteo de células viables bajo microscopio invertido. Se determinó la dosis letal media $\mathrm{DL}_{50}$.

\section{Análisis estadístico}

La digitación se hizo en bases de datos construidas en Excel versión 2003; la depuración y el procesamiento de la información en el programa StatGraphics Plus versión 2.0 y en el SPSS versión 12.0. Se calculó la dosis letal media $\mathrm{LD}_{50}$ y la concentración media inhibitoria $\mathrm{IC}_{50}$ utilizando el programa Probit.

\section{RESULTADOS}

El rendimiento de la mezcla de alcaloides obtenida a partir de la parte aérea de Ervatamia coronaria fue de $0.6 \%$. La monocapa de macrófagos $\mathrm{J} 774$ mostró que bajo las concentraciones mayores de alcaloides $(50 \mathrm{y}$ $100 \mu \mathrm{g} / \mathrm{mL}$ ) la morfología de los macrófagos fue más pequeña y redondeada, comparada con macrófagos con

un tratamiento de alcaloides a menor concentración. Por otro lado, en cuanto al tratamiento de adición de alcaloides una vez al día durante 3 días, se observó una disminución notable de células, especialmente con la más alta concentración: $100 \mu \mathrm{g} / \mathrm{mL}$.

\section{Comportamiento de la Infección en macrófagos J774. Actividad antiamastigote}

Las dos pruebas durante la infección, adición de alcaloides después de 1 hora de infección y 1 vez al día durante 3 días, mostraron diferencias entre ellos en cuanto a los índices de supervivencia del parásito e infección.

En la adición de la mezcla 1 hora post-infección se obtuvo, a manera general, una relación directa inversa entre concentración de alcaloides y porcentaje de infección, las mayores concentraciones presentaron los menores índices de infección y viceversa, excepto en la concentración $2(10 \mu \mathrm{g} / \mathrm{mL})$ que presentó la mayor proporción de infección (Figura 1). Estadísticamente existe una diferencia significativa entre el grupo control y cada uno de los tratamientos, el primero con proporción de la infección de 0.30 . K-W p $<0.05$ mostró que entre las mayores concentraciones y las menores hay una diferencia en la actividad leishmanicida, al igual que dentro de las concentraciones altas.

En cuanto a la infección resultante de la adición de alcaloides diariamente durante 3 días, las proporciones fueron similares en todas las concentraciones, excepto en la de $25 \mu \mathrm{g} / \mathrm{mL}$ cuyo valor fue el menor, correspondiente a 0.09. En este tratamiento, contradictoriamente, el valor más alto de infección correspondió a la mayor concentración de alcaloides. También existió en este protocolo una diferencia estadísticamente significativa, $\mathrm{p}<0.05$, de cada uno de los tratamientos con el grupo control (Figura 1). Al hacer las comparaciones entre grupos, no se registraron diferencias entre las concentraciones 1,2 y 3 , por el contrario la concentración 4 presentó diferencias con el resto de grupos. En el caso de las mayores concentraciones (5 y 6) no hubo diferencias al ser comparadas con las menores concentraciones ( 1 y 2$)$.

Los índices de supervivencia son muy similares al resultado de las proporciones de infección bajo ambos protocolos temporales. Aunque el comportamiento de la supervivencia (Figura 2) del parásito tratado 3 veces con la mezcla de alcaloides si mostró ciertas diferencias, comparado con la proporción de la infección. En la supervivencia del parásito no existieron diferencias entre las concentraciones 3 y 6 , ni tampoco 5 y 6 , contrariamente a lo que sucede en la proporción de infección. Las concentraciones más bajas presentaron diferencias en la supervivencia de Leishmania, pero no entre las mayores.

La diferencia entre ambos tratamientos es evidente, de las cuales la adición diaria por 3 días muestra valores 
menores de supervivencia del parásito. Un 47.2\% y $44.4 \%$ de supervivencia del amastigote se obtuvo a 1a menor concentración, $1 \mu \mathrm{g} / \mathrm{mL}$, a 1 hora y 72 horas postinfección de adicionar los alcaloides; mientras que a la mayor concentración, $100 \mu \mathrm{g} / \mathrm{mL}$, fue de 19.3 y $36 \%$ respectivamente (Figura 3).

\section{Ensayo de citotoxicidad en macrófagos $\mathbf{J 7 7 4}$}

Se evidenció una relación negativa dosisdependiente (Figura 4) sobre los macrófagos. Todos los porcentajes de supervivencia son valores altos, aún con altas concentraciones $(50$ y $100 \mu \mathrm{g} / \mathrm{mL}$ ) para la cual la viabilidad de los macrófagos sometidos a estos tratamientos correspondió a 61 y $54 \%$, respectivamente. Al comparar estadísticamente los grupos $(\mathrm{p}<0.5)$ las diferencias significativas se obtuvieron entre las 2 concentraciones menores.

\section{DISCUSIÓN}

En este trabajo se describe por primera vez la actividad leishmanicida de la mezcla de alcaloides de Ervatamia coronaria sobre amastigotes del parásito $L$. braziliensis.

Estudios similares han mostrado actividad contra otras especies del parásito. Delorenzi et al. (2001) demostraron que la coronaridina tiene actividad antipromastigote significativa, del $97 \%$ en una concentración de $12 \mu \mathrm{g} / \mathrm{mL}$ contra $L$. amazonensis. Extracto de Himatanthus sucuuba inhibió la supervivencia en $45.5 \%$ a $10 \mu \mathrm{g} / \mathrm{mL}$, mientras que a $100 \mu \mathrm{g} / \mathrm{mL}$ fue de $76 \%$ (Freire de Lima et al., 2000). Comparando estos porcentajes con el presente estudio se observa que la mezcla de Ervatamia coronaria presentó mejor eficacia, con inhibición de $67.6 \%$ a $10 \mu \mathrm{g} / \mathrm{mL} 1$ hora post-infección y de $83 \%$ en el tratamiento durante 3 días en la misma concentración.

Las diferencias entre estudios han sido frecuentes, aún sobre la misma especie de parásito y aún no se conoce el fenómeno que lo origine. Sin embargo, se atribuye a que, probablemente, existe una sensibilidad innata del parásito.

Aunque el mecanismo de acción leishmanicida de este tipo de alcaloides indólicos no se conoce aún (Staerk et al., 2000) es claro que existe una asociación con la estructura química y en especial, con la longitud del grupo de sustitución. Es muy probable que la estructura de otros tipos de alcaloides indólicos no estudiados, como el caso de los presentes en Ervatamia coronaria, influya de manera notable en la actividad sobre los parásitos. En los últimos años han surgido inferencias interesantes a partir de análisis de estructura del alcaloides/actividad leishmanicida. Del Rayo et al. (2002) han establecido que el estado de oxidación y la naturaleza de substitución de los átomos del nitrógeno son aspectos importantes para la actividad leishmanicida y determinan la bioactividad del alcaloide. La cuaternización de uno o más átomos de nitrógeno resulta en la pérdida de actividad leishmanicida, la suma de un grupo fenólico hidroxilo puede aumentar la actividad, al igual que diferencias en la estequiometría. Staerk et al. (2000) sugieren que la estructura relativamente planar tetracíclica confiere la actividad antiparasitaria de estos compuestos. Los alcaloides de la especie Ervatamia coronaria son de tipo indólico y bisindólico, los cuales carecen de cuaternización en sus nitrógenos y presentan la estructura relativamente planar.

Además de las diferencias en cuanto a eficacia de un compuesto en particular, es interesante resaltar que existen también diferencias al comparar evaluaciones de un extracto o la mezcla y un compuesto aislado. En general se observa una actividad leishmanicida mayor en el extracto que en el compuesto particular. En el trabajo de Delorenzi et al. (2001) se obtuvo para la forma promastigota, una inhibición de la supervivencia del parásito de $97 \%$ con la adición de $12.5 \mu \mathrm{g} / \mathrm{mL}$ de coronaridina, mientras que la fracción de cloroformo a la misma concentración inhibió el 65\% del crecimiento. Estas diferencias pueden ser explicadas gracias a que en la fracción de cloroformo existen otros compuestos, que seguramente presentan un efecto sinérgico; mientras que la coronaridina aunque logre controlar el parásito, evidentemente no es el único elemento en el extracto que juega un papel en este proceso.

Los índices de infección y supervivencia aunque no mostraron una tendencia lineal decreciente en términos puntuales, en una visión general si se detecta la relación inversa dosis-dependiente entre concentración y comportamiento del parásito. Los casos en que se rompe esta tendencia son los correspondientes a la concentración $2(10 \mu \mathrm{g} / \mathrm{mL})$ de la adición de alcaloides 1 hora post-infección, y las concentraciones más altas (50 y $100 \mu \mathrm{g} / \mathrm{mL}$ ) de adición durante 3 días.

En el caso de la concentración 2, que mostró una proporción de infección de 0.24 frente a 0.16 de la concentración 1 -estadísticamente diferentes $\mathrm{p}<0.05$ - el elevado valor de infección puede explicarse en la amplia variabilidad que mostraron los datos tomados, como lo muestra la Figura 1. De modo que valores extremos aumentaron el índice de proporción de la infección y así mismo el de supervivencia.

En cuanto a las altas proporciones de infección que se mantuvieron en las mayores concentraciones evaluadas durante 3 días (50 y $100 \mu \mathrm{g} / \mathrm{mL}$ ), pueden ser explicadas con base a la población celular que es esencial en el cálculo de los índices de porcentaje y proporción de la infección. Se sugiere que estas concentraciones afectan directamente el macrófago y su ciclo de división celular, por lo que se presentó un número más reducido de células, de las cuales algunas estaban infectadas por el parásito y así los índices de infección se vieron aumentados respecto a las demás concentraciones. En el caso de este mismo tratamiento 1 hora post-infección 


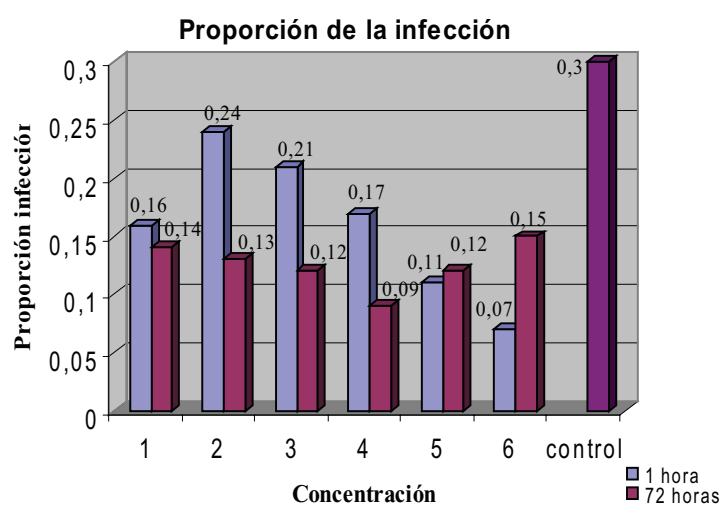

Figura 1. Figura de la proporción de infección en el grupo control y en los tratamientos de adición de mezcla de alcaloides 1hora post-infección y diariamente durante 3 días.

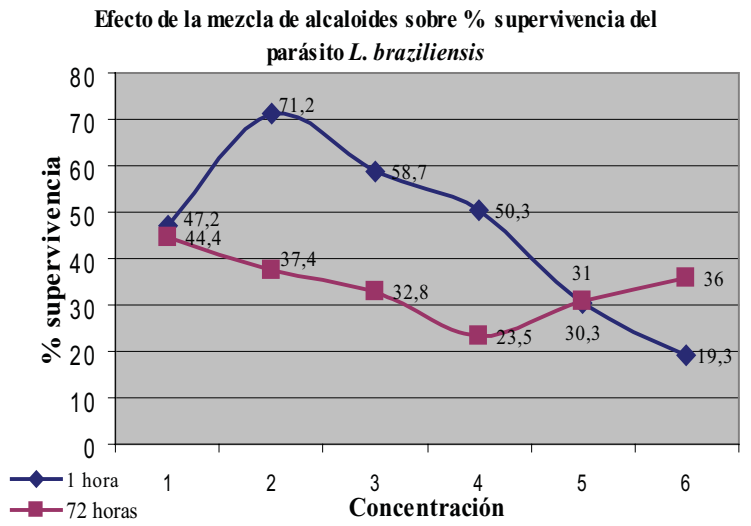

Figura 3. Porcentaje de supervivencia del parásito intracelular en las 6 concentraciones evaluadas.

el número celular no se vio igualmente afectado puesto que se adicionó el compuesto sólo $1 \mathrm{vez}$, de manera que las células no se encontraron igualmente afectadas en comparación a la adición de la concentración, además alta, durante 3 días.

La $\mathrm{IC}_{50}$ obtenida mostró valores de 2.6 y 12.4 $\mu \mathrm{g} / \mathrm{mL}$ para adición del compuesto 1 hora post-infección y por 3 días, respectivamente. Muchos estudios muestran valores menores, especialmente para alcaloides aislados (Del Rayo et al., 2002; Staerk et al., 2000; Keawpradub et al., 1999). Sin embargo, hay similitudes con trabajos de Delorenzi et al. (2001) cuyo valor para el tratamiento después de 1 hora de infección es el mismo contra $L$. amazonensis, a partir de alcaloides de Peschiera australis, también Apocynaceae. Para la evaluación de la adición durante 3 días, este autor obtuvo un valor menor (1.25). También es necesario tener en cuenta que lo obtenido en el presente estudio, presenta actividad antiparasitaria más eficaz que algunas drogas, como stiboglucanato y allopurinol (5.6 y $8.8 \mu \mathrm{g} / \mathrm{mL}$, respectivamente).

Se han reportado (Marchán et al., 2000)

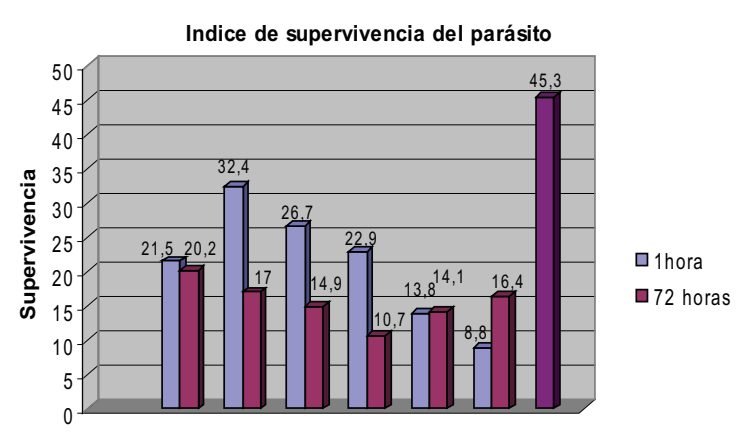

Figura 2. Índice de supervivencia del parásito a los 2 tiempos de adición de alcaloides.

Viabilidad de macrófagos $\mathbf{J 7 7 4}$ bajo varias concentraciones de mezcla de alcaloides de Ervatamia coronaria

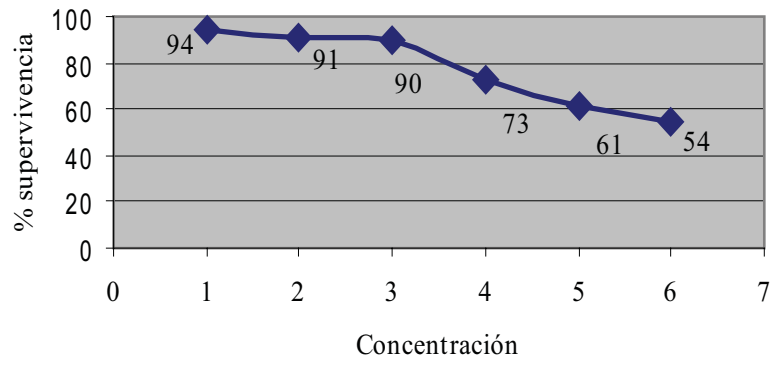

Figura 4. Porcentaje de supervivencia de macrófagos J774 bajo 6 diferentes concentraciones.

cambios morfológicos en los parásitos, obteniendo incremento en el volumen celular asociado con daños en el contenido celular y la destrucción de organelos, así como separación de la membrana y el flagelo y vacuolización progresiva, aún en la concentración más baja, que corresponde a $4 \mu \mathrm{g} / \mathrm{mL}$. De otro lado, la berberina, alcaloide ampliamente estudiado, inhibe la incorporación de adenina, uracilo y timidina en los ácidos nucleicos, y de leucina en las proteínas de los amastigotes de L. donovani. Lo anterior muestra que los compuestos tipo alcaloide ejercen un efecto inhibitorio sobre los parásitos asociados a profundos cambios ultraestructurales, o bien sobre la biosíntesis de macromoléculas, fenómenos que probablemente se produzcan también con la mezcla de alcaloides de Ervatamia coronaria sobre L. braziliensis.

Los porcentajes de viabilidad sobre macrófagos en las diferentes concentraciones muestran valores altos hasta lo correspondiente a $25 \mu \mathrm{g} / \mathrm{mL}$, cuyo valor es de $73 \%$. Freire de Lima et al. (2000), a concentración de $20 \mu \mathrm{g} / \mathrm{mL}$ de extracto de Himatanthus sucuuba no 
observaron efecto citotóxico, con un porcentaje de supervivencia de $85 \%$, mientras que en el presente estudio bajo esa concentración fue de $90 \%$.

Soares etal.(2003) evaluaron la citotoxicidad del extracto obtenido a partir de la especie Tabernaemontana catharinensis, de la cual se cuantificaron mediante cromatografía de gas los alcaloides coronaridina y voacristina, demostrando que no presentaron efectos tóxicos en macrófagos murinos de ratón. En estudios realizados con varios alcaloides (Marshall et al., 1994), se encontró que el más citotóxico, berbamina, con $\mathrm{IC}_{50}$ de $17.8 \mu \mathrm{M}$ fue casi 2000 veces menos potente que la droga control, glucantime.

Los cambios morfológicos vistos en los macrófagos bajo las más elevadas concentraciones de alcaloides se relacionan con los datos obtenidos en el ensayo de citotoxicidad. Delorenzi et al. (2001) evaluaron la morfología de macrófagos con la fracción de cloroformo de Peschiera australis, que contienen alcaloides indólicos. Macrófagos sin infectar tratados con $20 \mu \mathrm{g} / \mathrm{mL}$ durante 24 horas no mostraron diferencias con el grupo control. Las células presentaron núcleo definido, retículo endoplásmico, aparato de Golgi y mitocondrias con sus membranas claramente definidas. Mientras que aquellos tratados con la misma concentración de coronaridina, en la mitocondria se observó aumento de tamaño, llena de un material amorfo, aunque comparada con la mitocondria del parásito bajo el mismo tratamiento se encontró bien conservada.

\section{AGRADECIMIENTOS}

A los miembros de GIFUJ de la Universidad Javeriana y del Laboratorio de Entomología, Biología Celular y Genética de La Universidad de la Salle por la colaboración y financiación en el trabajo.

\section{REFERENCIAS}

Agra MF, França PF, Barbosa-Filho JM 2007. Synopsis of the plants known as medicinal and poisonous in Northeast of Brazil. Rev Bras Farmacogn 17: 114140

Berman JD 1997. Human Leishmaniosis: clinical, diagnostic, and chemotherapeutic developments in the last 10 years. Clin Infect Dis 24: 684-703.

Del Rayo M, Phillipson JD, Croft S, Rock P, Marshall S, Schiff PL 2002. In vitro activity of Triclisia patens and some bisbenzylisoquinoline alkaloids against Leishmania donovani and Trypanosoma brucei brucei. Phytother Res 16: 432-436.

Delorenzi JC, Attias M, Gattass C, Andrade M, Rezende C, Da Cunha A, Henriques A, Bou-Habib D, Saraiva E 2001. Antileishmanial activity of an indole alkaloid from Peschiera australis. Antimicrob Agents Chemother 45: 1349-1354.

Freire-de-Lima L, Delorenzi J, Okada LH, Saraiva EMB 2000. Potential leishmanicide activity of Himatanthus sucuuba (Apocyanceae). Mem I Oswaldo. Cruz 95
(Supl. 2): 312.

Keawpradub N, Kirby GC, Steele JCP, Houghton PJ 1999. Antiplasmodial activity of extracts and alkaloids of three Alstonia species from Thailand. Planta Med 65: 690-694.

Marchán E, Arrieche D, Henriquez W, Crescente O 2000. Efecto in vitro de una sustancia alcaloidea aislada de Amphimedon virides (Porifera) sobre promastigotes de Leishmania mexicana. Rev Biol Trop 48 (Supl. 1): 31-38.

Marquez R 1999. Alcaloides en la semilla de Tabernaemontana coronaria (Apocynaceae) y evaluación de la actividad mutagénica. Bogotá. Trabajo de grado Postgrado en Biología con énfasis en fitoquímica. Microficha. Pontificia Universidad Javeriana.

Marshall S, Russell P, Wright C, Anderson M, Phillipson D, Kirby G, Warhust D, Svhiff P 1994. In vitro antiplasmodial, antiamoebic and cytotoxic activities of a series of bisbenzylisoquinoline alkaloids. Antimicrob Agents Chemother 38: 96-103.

Piloto IC, Campana M, Machado GM, Leon LL, Gobby L, Bissoli LH 2004. Anti-leishmanial activity of alkaloid extract from Aspidosperma ramiflorum. Mem I Oswaldo Cruz 99: 325- 327.

Quintans-Júnior LJ, Silva DA, Siqueira JS, Souza MFV, Almeida RN, Silva-Júnior RGC 2007. Anticonvulsant properties of the total alkaloid fraction of Rauvolfia ligustrina Roem. et Schult. in male mice. Rev Bras Farmacogn 17: 176-180.

Rocha LG, Almeida JRGS, Macedo RO, Barbosa-Filho JM 2005. A review of natural products with antileishmanial activity. Phytomedicine 12: 514535.

Soares DC, Pereira CG, Meireles MA, Saraiva EMB 2003. AntiLeishmania (L.) amazonensis activity of supercritical $\mathrm{CO}_{2}^{+}$ethanol extracts from Tabernaemontana catharinensis. Rev Inst Med Trop. Sao Paulo 45 (Supl. 13): 110.

Souza WM, Brehmer F, Nakao LS, Stinghen AEM, Santos CAM 2007. Ação da uleína sobre a produção de óxido nítrico em células RAEC e B16F10. Rev Bras Farmacogn 17: 191-196.

Staerk D, Lemmich E, Christensen J, Kharazmi A, Olsen CE, Jeroszewski J 2000. Leishmanicidal, antiplasmodial and cytotoxic activity of indole alkaloids from Corynanthe pachyceras. Planta Med 66: 531-536. 